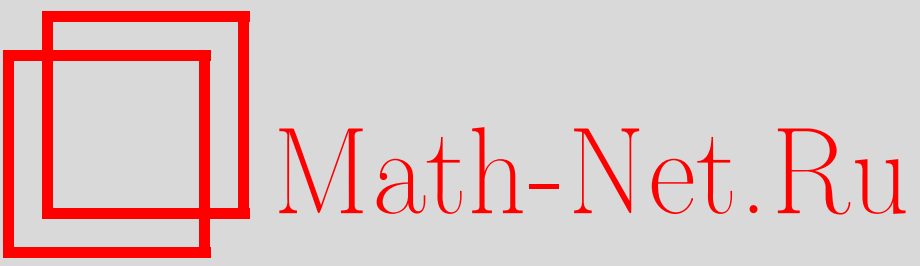

А. А. Замятин, А. А. Ямбарцев, Классификация двух взаимодействующих цепочек символов, УМH, 2001, том 56, выпуск 3, 165-166

DOI: https://doi.org/10.4213/rm404

Использование Общероссийского математического портала Math-Net.Ru подразумевает, что вы прочитали и согласны с пользовательским соглашением

http://www.mathnet.ru/rus/agreement

Параметры загрузки:

IP: 52.23 .180 .231

26 апреля 2023 г., 15:45:20 


\title{
КЛАССИФИКАЦИЯ ДВУХ ВЗАИМОДЕЙСТВУЮЩИХ ЦЕПОЧЕК СИМВОЛОВ
}

\author{
А. А. ЗАМЯтИн, А. А. ЯмБАРцЕВ
}

1. Конечным словом $\alpha=x_{1} x_{2} \ldots x_{n}$ назьвается конечная последовательность символов $x_{i}$ из некоторого алфавита $\mathscr{S}=\{1,2, \ldots, r\}$. Обозначим через $|\alpha|$ длину слова $\alpha$. Пусть $\mathscr{A}$ - множество всех конечных слов, включая пустое. Композицией двух слов $\alpha=x_{1} x_{2} \ldots x_{n}$ и $\beta=y_{1} y_{2} \ldots y_{m}$ назовем слово $\alpha \beta=x_{1} x_{2} \ldots x_{n+m}$, где $x_{n+1}=y_{1}, \ldots, x_{n+m}=y_{m}$.

Последовательность $\alpha=\ldots y_{n-1} y_{n}$ символов из алфавита назовем полубесконечным словом. Его можно определить парой $(n, \xi), n \in \mathbb{Z}$ и $\xi=\ldots x_{-1} x_{0}$, где $x_{0}=y_{n}, x_{-1}=y_{n-1}, \ldots-$ последовательность символов с выделенной нумерацией. Такую последовательность будем называть средой. Множество всех полубесконечных слов обозначим через $\mathscr{A}_{\infty}$, а совокупность всевозможных сред - через $\mathscr{E} \infty$. Аналогично можно определить композицию $\alpha \beta$ полубесконечного слова $\alpha=\ldots x_{n-1} x_{n}$ с конечным словом $\beta=y_{1} \ldots y_{m}$.

2. Определим однородную цепь Маркова $\mathscr{L}_{\infty}$ на множестве состояний $\mathscr{A}_{\infty}^{2}=\mathscr{A}_{\infty} \times \mathscr{A}_{\infty}$. Обозначим $\eta(t)=\left(\eta_{1}(t), \eta_{2}(t)\right)$ состояние цепи в момент времени $t$. Забиксируем некоторое натуральное $d$. Переходные вероятности $\mathrm{P}\{\eta(t+1)=\beta \mid \eta(t)=\alpha\} \neq 0$ только в том случае, если $\beta=\rho \theta, \alpha=\rho \gamma$, где $\rho \in \mathscr{A}_{\infty}^{2}$ и $\gamma=\left(\gamma_{1}, \gamma_{2}\right), \theta=\left(\theta_{1}, \theta_{2}\right) \in \mathscr{A}^{2}=\mathscr{A} \times \mathscr{A}$ такие, что $\left|\gamma_{k}\right|=d,\left|\theta_{k}\right| \leqslant 2 d(k=1,2)$. Здесь для любых пар $\alpha=\left(\alpha_{1}, \alpha_{2}\right) \in \mathscr{A}_{\infty}^{2}$ и $\beta=\left(\beta_{1}, \beta_{2}\right) \in \mathscr{A}^{2} \alpha \beta=\left(\alpha_{1} \beta_{1}, \alpha_{2} \beta_{2}\right) \in \mathscr{A}_{\infty}^{2}$. Предположим также, что вероятности перехода $q(\gamma, \theta)=\mathrm{P}\{\eta(t+1)=\rho \theta \mid \eta(t)=\rho \gamma\}$ зависят только от $\gamma, \theta$. Обозначим через $\mathscr{P}$ множество вероятностных мер на $\mathscr{E}_{\infty}^{2}=\mathscr{E}_{\infty} \times \mathscr{E}_{\infty}$.

Для $\alpha \in \mathscr{A}_{\infty}$ выражение $[\alpha]_{n}=\gamma$, где $|\gamma|=n$, означает, что $\alpha=\rho \gamma$ для некоторого $\rho \in \mathscr{A}_{\infty}$. Корреляционные функции

$$
p_{t}\left(\gamma_{1}, \gamma_{2}\right)=\operatorname{P}\left\{\left[\eta_{1}(t)\right]_{\left|\gamma_{1}\right|}=\gamma_{1},\left[\eta_{2}(t)\right]_{\left|\gamma_{2}\right|}=\gamma_{2}\right\},
$$

где $\gamma_{k} \in \mathscr{A}$, однозначно определяют меру $\mu(t) \in \mathscr{P}$. Меру $\mu$ назовем инвариантной мерой для цепи $\mathscr{L}_{\infty}$, если $\mu(t)=\mu$ для любого $t$. Инвариантная мера $\mu$ называется әкстремальной, если она не представима в виде выпуклой линейной комбинации других инвариантных мер. Пусть $\mu$ - инвариантная мера и $p_{\mu}(\gamma), \gamma \in \mathscr{A}^{2},-$ ее корреляционные функции. Каждой инвариантной $\mu$ сопоставим вектор сноса $v(\mu)$ с компонентами

$$
v_{i}(\mu)=\sum_{\gamma_{1}, \gamma_{2}} \sum_{\theta_{1}, \theta_{2}}\left(\left|\theta_{i}\right|-d\right) q\left(\left(\gamma_{1}, \gamma_{2}\right),\left(\theta_{1}, \theta_{2}\right)\right) p_{\mu}\left(\gamma_{1}, \gamma_{2}\right)
$$

где $\left|\gamma_{k}\right|=d,\left|\theta_{k}\right| \leqslant 2 d(k=1,2)$, и вектор знаков

$$
\operatorname{sign}(\mu)=\left(\operatorname{sign}\left(v_{1}(\mu)\right), \operatorname{sign}\left(v_{2}(\mu)\right)\right), \quad \operatorname{sign}\left(v_{i}(\mu)\right)=-, 0,+.
$$

Инвариантная мера $\mu$ называется мерой типа $\left(\sigma_{1}, \sigma_{2}\right)$, если $\operatorname{sign}(\mu)=\left(\sigma_{1}, \sigma_{2}\right)$, где $\sigma_{i}=-, 0,+$.

3. В [1] приведен пример сосуществования мер типов $(-,+)$ и $(-,-)$. Здесь мы построим пример цепи $\mathscr{L}_{\infty}$, которая имеет инвариантные меры типов $(-,+),(+,-),(-,-)$. Пусть $\mathscr{S}=$ $\{1,2\}$ и $d=1$. Предположим, что вероятность каждого из переходов: $(1,1) \rightarrow(1, \varnothing),(2,2) \rightarrow$ $(\varnothing, 2),(1,2) \rightarrow(\varnothing, \varnothing),(2,1) \rightarrow(\varnothing, \varnothing)$ больше, чем $1-\varepsilon$.

ПРЕДЛОЖЕНИЕ. При достаточно малом $\varepsilon>0$ сосуществуют инвариантные меры muпов $(-,+),(+,-)$ и $(-,-)$.

Работа выполнена при поддержке Российского фонда фундаментальных исследований (грант № 98-01-00653). 
4. Основной объект нашего внимания - цепь Маркова $\mathscr{L}$ на множестве состояний $\mathscr{A}^{2}$. Пусть $\Lambda \subseteq\{1,2\}$ и $F(\Lambda)=\left\{\alpha=\left(\alpha_{1}, \alpha_{2}\right):\left|\alpha_{i}\right| \geqslant d, i \in \Lambda ;\left|\alpha_{i}\right|<d, i \notin \Lambda\right\} . F(1), F(2)$ будем называть гранями. Пусть $\alpha(t)=\left(\alpha_{1}(t), \alpha_{2}(t)\right)$ - состояние цепи $\mathscr{L}$ в момент $t$. Зададим вероятности перехода $p_{\alpha \beta}=\mathrm{P}\{\alpha(t+1)=\beta \mid \alpha(t)=\alpha\}$ следующим образом. Если $\alpha \in F(1,2)$, то вероятности переходов такие же, как в случае цепи $\mathscr{L}_{\infty}$. Если $\alpha \in F(1)$, то переходные вероятности $p_{\left(\alpha_{1}, \alpha_{2}\right),\left(\beta_{1}, \beta_{2}\right)} \neq 0$ толшко в том случае, если $\alpha_{1}=\rho_{1} \gamma_{1}, \beta_{1}=\rho_{1} \theta_{1}$, где $\left|\gamma_{1}\right|=d$, $\left|\theta_{1}\right| \leqslant 2 d,\left|\beta_{2}\right| \leqslant d$. Положим $q_{1}\left(\left(\gamma_{1}, \alpha_{2}\right),\left(\theta_{1}, \beta_{2}\right)\right)=p_{\left(\rho_{1} \gamma_{1}, \alpha_{2}\right),\left(\rho_{1} \theta_{1}, \beta_{2}\right)}$. Аналогично определяются переходные вероятности $q_{2}\left(\left(\alpha_{1}, \gamma_{2}\right),\left(\beta_{1}, \theta_{2}\right)\right)$ на грани $F(2)$. Относительно переходных вероятностей $p_{\alpha \beta}$ для $\alpha \in F(\varnothing)$ предположим ограниченность скачков: длина каждого слова за один шаг изменяется не более чем на $d$. Мы будем предполагать также, что все определенные вьше переходные вероятности ненулевые.

5. С гранями $F(1), F(2)$ свяжем индуцированные марковские цепи $\mathscr{L}_{1}, \mathscr{L}_{2}$ на множествах $\mathscr{D}_{1}=\mathscr{A}_{\infty} \times \mathscr{A}_{\text {и }} \mathscr{D}_{2}=\mathscr{A} \times \mathscr{A}_{\infty}$ соответственно. Пусть $\zeta^{(\Lambda)}(t)=\left(\zeta_{1}^{(\Lambda)}(t), \zeta_{2}^{(\Lambda)}(t)\right) \in \mathscr{D}_{\Lambda}-$ состояние индуцированной цепи $\mathscr{L}_{\Lambda}$ в момент $t$. Эволюция цепи $\mathscr{L}_{\Lambda}$ определяется вероятностями перехода $q(\gamma, \theta), q_{\Lambda}(\gamma, \theta)$.

Пусть $\widetilde{\mathscr{D}}_{1}=\mathscr{E}_{\infty} \times \mathscr{A}$, и $\widetilde{\mathscr{D}}_{2}=\mathscr{A} \times \mathscr{E}_{\infty}$. Обозначим $\mathscr{P}_{\Lambda}$ множество вероятностных мер на пространстве $\widetilde{D}_{\Lambda}$. Корреляционные функции цепей $\mathscr{L}_{1}$ и $\mathscr{L}_{2}$

$$
\begin{aligned}
& p_{t}^{(1)}\left(\gamma_{1}, \gamma_{2}\right)=\mathrm{P}\left\{\left[\zeta_{1}^{(1)}(t)\right]_{\left|\gamma_{1}\right|}=\gamma_{1}, \zeta_{2}^{(1)}(t)=\gamma_{2}\right\}, \\
& p_{t}^{(2)}\left(\gamma_{1}, \gamma_{2}\right)=\mathrm{P}\left\{\zeta_{1}^{(2)}(t)=\gamma_{1},\left[\zeta_{2}^{(2)}(t)\right]_{\left|\gamma_{2}\right|}=\gamma_{2}\right\}
\end{aligned}
$$

для любого $t$ однозначно определяют меры $\mu^{(1)}(t) \in \mathscr{P}_{1}, \mu^{(2)}(t) \in \mathscr{P}_{2}$. Меру $\mu^{(\Lambda)}$ назовем инвариантной мерой для цепи $\mathscr{L}_{\Lambda}$, если $\mu^{(\Lambda)}(t)=\mu^{(\Lambda)}$ для любого $t$. Пусть $\mathscr{M}_{\Lambda} \subset \mathscr{P}_{\Lambda}-$ множество инвариантных мер цепи $\mathscr{L}_{\Lambda}$. Грань $F(\Lambda)$ назьвается әргодической, если $\mathscr{M}_{\Lambda} \neq \varnothing$. Каждой инвариантной мере $\nu$ цепи $\mathscr{L}_{\Lambda}$ сопоставим вектор сноса

$$
\begin{aligned}
v^{(\Lambda)}(\nu)= & \sum_{\gamma_{k}, \theta_{k}}\left(\left|\theta_{\Lambda}\right|-d\right) q\left(\left(\gamma_{1}, \gamma_{2}\right),\left(\theta_{1}, \theta_{2}\right)\right) \sum_{\beta \in \mathscr{A}} p_{\nu}^{(\Lambda)}\left(\gamma_{1}, \beta \gamma_{2}\right) \\
& +\sum_{\gamma_{k}, \theta_{k}}\left(\left|\theta_{\Lambda}\right|-d\right) q_{\Lambda}\left(\left(\gamma_{1}, \gamma_{2}\right),\left(\theta_{1}, \theta_{2}\right)\right) p_{\nu}^{(\Lambda)}\left(\gamma_{1}, \varnothing\right)
\end{aligned}
$$

и знак

$$
\operatorname{sign}(\nu)=\operatorname{sign}\left(v^{\Lambda}(\nu)\right)
$$

Инвариантная мера $\nu$ назьвается мерой типа $(\sigma)$, если $\operatorname{sign}(\nu)=\sigma$, где $\sigma=-, 0,+$. Мы предполагаем, что $\operatorname{sign}(\nu) \neq 0$ для всех $\nu \subset \mathscr{M}_{\Lambda}, \Lambda=1,2$.

ТЕОРема 1. 1. Если марковская цепь $\mathscr{L}_{\infty}$ имеет әкстремальную инвариантную меру mипа $(+,+)$, тогда чепь $\mathscr{L}$ транзиентна.

2. Если для некоторой эргодической грани существует инвариантная мера типа (+), тогда марковская иепь $\mathscr{L}$ транзиентна.

ТеОРема 2. Предположим, что все инвариантные меры иепи $\mathscr{L}_{\infty}$ имеют один и mот же тип: $(-,-),(-,+)$ или $(+,-)$. Тогда существует по крайней мере одна әргодическая грань, и если для любой эргодической грани существует хотя бь одна инвариантная мера типа (-), то цепь Маркова $\mathscr{L}$ әргодична.

Первое утверждение теоремы 1 доказано в [2].

\section{СПИСОК ЛИТЕРАТУРЫ}

[1] В. А. Малышев // УМН. 1997. Т. 52. №2. С. 59-86. [2] А. А. Замятин, А. А. Ямбарцев // Фунд. и прикл. матем. 1996. Т. 2. № 4. С. 1029-1043. 